\title{
On Total and Edge-colouring of Proper Circular-arc Graphs
}

\author{
João Pedro W. Bernardi ${ }^{1,2 *}$, Sheila M. de Almeida ${ }^{3 *}$, Leandro M. Zatesko ${ }^{1,2 * \dagger}$ \\ ${ }^{1}$ Federal University of Fronteira Sul, Chapecó, Brazil \\ ${ }^{2}$ Federal University of Paraná, Curitiba, Brazil \\ ${ }^{3}$ Federal University of Technology — Paraná, Ponta Grossa, Brazil \\ \{winckler, leandro.zatesko\} dufpr.br, sheilaalmeida@utfpr.edu.br
}

\begin{abstract}
Deciding if a graph is $\Delta$-edge-colourable (resp. $(\Delta+1)$-total colourable), although it is an NP-complete problem for graphs in general, is polynomially solvable for interval graphs of odd (resp. even) maximum degree $\Delta$. An interesting superclass of the proper interval graphs are the proper circular-arc graphs, for which we suspect that $\Delta$-edge-colourability is linear-time decidable. This work presents sufficient conditions for $\Delta$-edge-colourability, $(\Delta+1)$-total colourability, and $(\Delta+2)$-total colourability of proper circular-arc graphs. Our proofs are constructive and yield polynomial-time algorithms.
\end{abstract}

\section{Introduction}

The chromatic index and the total chromatic number of a graph $G$ with maximum degree $\Delta$ clearly satisfy $\chi^{\prime}(G) \geq \Delta$ and $\chi^{\prime \prime}(G) \geq \Delta+1$ (see definitions in the sequel). Also, $\chi^{\prime}(G) \leq \Delta+1$ [Vizing 1964], and the Total Colouring Conjecture states that $\chi^{\prime \prime}(G) \leq$ $\Delta+2$ [Behzad 1965, Vizing 1968]. A graph $G$ is Class 1 if $\chi^{\prime}(G)=\Delta$, or Class 2 otherwise. Since no graph with $\chi^{\prime \prime}(G) \geq \Delta+3$ is known, graphs with $\chi^{\prime \prime}(G)=\Delta+1$ have been called Type 1 , and those with $\chi^{\prime \prime}(G)=\Delta+2$ Type 2. Deciding if $G$ is Class 1 and deciding if $G$ is Type 1 are NP-complete problems [Holyer 1981, Sánchez-Arroyo 1989].

The classes of the unit and the proper interval graphs are the same [Roberts 1969], but the classes of the unit and the proper circular-arc graphs are not (see Figure 1). The
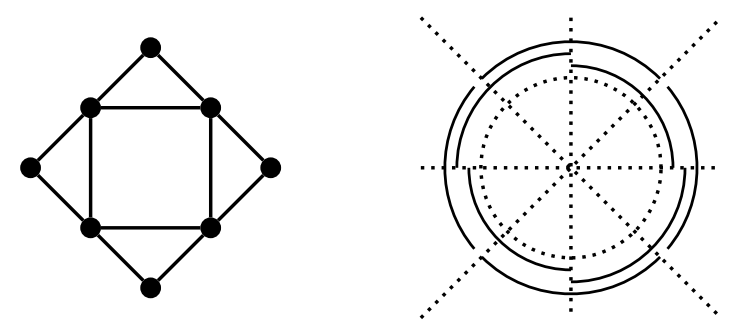

Figure 1. A proper non-unit circular-arc graph with a corresponding arc model

Total Colouring Conjecture holds for proper interval graphs, often referred to as indifference graphs, which are Class 1 when they have odd $\Delta$, and Type 1 when $\Delta$ is even [Figueiredo et al. 1997]. For edge-colouring of indifference graphs with even $\Delta$ or total colouring of these graphs with odd $\Delta$, partial results are known [Figueiredo et al. 2003,

*Partially supported by CNPq, Proc. 428941/2016-8.

${ }^{\dagger}$ Partially supported by UFFS, Proc. 23205.001243/2016-30. 
Campos et al. 2012]. Recall that interval graphs are perfect graphs and, thus, admit polynomial-time vertex-colouring algorithms [Grötschel et al. 1981], in contrast to vertex-colouring of circular-arc graphs, which is NP-hard [Garey et al. 1980]. To the best of our knowledge, there is no published work on total or edge-colouring of circular-arc graphs.

Let $G$ be an $n$-vertex proper circular-arc graph. We show that if $n \equiv 0(\bmod (\Delta+$ $1)$ ), or if $G$ has a maximal clique of size 2 and $n \not k k(\bmod (\Delta+1))$ for all $k \in\{1, \Delta\}$, then: $\chi^{\prime}(G)=\Delta$ and $\chi^{\prime \prime}(G) \leq \Delta+2$ if $\Delta$ is odd; $\chi^{\prime \prime}(G)=\Delta+1$ if $\Delta$ is even. This implies that the Total Colouring Conjecture holds for the class of all such graphs.

This paper is organised as follows: the remaining of this section provides further definitions and discusses other related results in the literature; Section 2 presents our results; at last, Section 3 makes remarks on edge-colouring proper circular-arc graphs.

\section{Preliminary definitions and other related results}

This work deals only with simple graphs, referred to simply as graphs. Usual terms concerning graph-theoretical concepts follow their definitions and notation in the literature. In particular, the degree of a vertex $u$ in a graph $G$, the set of neighbours of $u$ in $G$, and the set of the edges incident to $u$ in $G$ are denoted by $d_{G}(u), N_{G}(u)$, and $\partial_{G}(u)$, respectively.

Let $G=(V, E)$ be a graph and $\mathscr{C}$ a set of $t$ colours. A function with $\mathscr{C}$ as its codomain is: a t-edge-colouring if its domain is $E$ and it is injective in $\partial_{G}(u)$ for all $u \in V$; a t-total colouring if its domain is $V \cup E$ and it is injective in $\partial_{G}(u) \cup\{u\}$ for all $u \in V$ and injective in $\{u, v\}$ for all $u v \in E$. In a total or edge-colouring, we say that a colour is missing at a vertex $u$ if it is not assigned to $u$ or to any edge incident to $u$. The chromatic index (denoted by $\chi^{\prime}(G)$ ) and the total chromatic number (denoted by $\chi^{\prime \prime}(G)$ ) of $G$ are the least $t$ for which $G$ is $t$-edge-colourable and $t$-total colourable, respectively.

An $n$-vertex graph with more than $\Delta\lfloor n / 2\rfloor$ edges (thus Class 2, since at most $\lfloor n / 2\rfloor$ edges can be coloured the same) is said to be overfull. It is conjectured that every graph $G$ with $\Delta>n / 3$ is Class 2 if and only if it is subgraph-overfull (shortly, SO), i.e. if $G$ has an overfull subgraph with the same maximum degree [Hilton and Johnson 1987].

The complete graph $K_{n}$ is: Class 1 and Type 2 if $n$ is even; Class 2 and Type 1 if $n$ is odd [Behzad et al. 1967]. Let $V\left(K_{n}\right):=\{0, \ldots, n-1\}$ and let even $(n)$ be 1 if $n$ is even or 0 otherwise. We call the canonical total and edge-colourings of the $K_{n}$ the functions $\varphi_{\text {edge }}^{\text {even }}, \varphi_{\text {edge }}^{\text {odd }}$, and $\varphi_{\text {total }}$ given by: $\varphi_{\text {edge }}^{\text {even }}(u v):=(u+v) \bmod (n-1)$, if neither $u$ nor $v$ is $n-1 ; \varphi_{\text {edge }}^{\text {even }}(u v):=(2 u) \bmod (n-1)$, if $v=n-1 ; \varphi_{\text {edge }}^{\text {odd }}(u v)=\varphi_{\text {total }}(u v):=$ $(u+v) \bmod (n+\operatorname{even}(n)) ; \varphi_{\text {total }}(u):=(2 u) \bmod (n+\operatorname{even}(n))$.

A circular-arc graph $G$ is the intersection graph of a finite set $S$ of arcs of a circle, in which case $S$ is an arc model corresponding to $G$. Furthermore, $G$ is said to be: proper, if there is a corresponding arc model wherein no arc properly contains another; a unit circular-arc graph, if there is a model wherein all the arcs have equal length. The vertices of a proper circular-arc graph admit a proper circular-arc order, i.e. a circular order in which vertices belonging to the same maximal clique appear consecutively. Homonymous terms are defined for interval graphs analogously, but being $S$ a set of intervals on the real line and the proper interval (or indifference) order a linear order. Interval and circular-arc graphs can be recognised in linear time [Booth and Lueker 1976, McConnell 2003].

A pullback from $G_{1}=\left(V_{1}, E_{1}\right)$ to $G_{2}=\left(V_{2}, E_{2}\right)$ is a homeomorphism $\pi: V_{1} \rightarrow$ 
$V_{2}$ (i.e. $\pi(u) \pi(v) \in E_{2}$ for all $u v \in E_{1}$ ) injective in $N_{G_{1}}(u) \cup\{u\}$ for all $u \in V_{1}$. If such a pullback exists and $G_{2}$ has a: $t$-edge-colouring $\varphi$, then a $t$-edge-colouring for $G_{1}$ can be given by $\psi(u v):=\varphi(\pi(u) \pi(v))$; $t$-total colouring $\varphi$, then a $t$-total colouring for $G_{1}$ can be given by $\psi(u v):=\varphi(\pi(u) \pi(v))$ and $\psi(u):=\varphi(\pi(u))$ [Figueiredo et al. 1997].

\section{Results}

Throughout this section, let $G$ be an $n$-vertex proper circular-arc graph. Remark that when we say that $G$ is $\Delta+2$-total colourable, it does not mean that $G$ is Type 2 .

Theorem 1. If $n \equiv 0(\bmod (\Delta+1))$, then $G$ is: Class 1 and $(\Delta+2)$-total colourable if $\Delta$ is odd; Type 1 if $\Delta$ is even.

Proof. It suffices to show that if $n \equiv 0(\bmod (\Delta+1))$, then there is a pullback from $G$ to the $K_{\Delta+1}$. Let $\sigma:=u_{0}, \ldots, u_{n-1}$ be a proper circular-arc order of $G$ and $0, \ldots, \Delta$ be the vertices of the $K_{\Delta+1}$. Assume, by the sake of contradiction, that the function $\pi: V(G) \rightarrow V\left(K_{\Delta+1}\right)$ defined by $\pi\left(u_{i}\right):=i \bmod (\Delta+1)$ is not a pullback from $G$ to the $K_{\Delta+1}$. As $\pi$ is clearly a homeomorphism, there must be two distinct vertices $v_{1}$ and $v_{2}$ in $V(G)$ which have a neighbour $w$ in common and satisfy $\pi\left(v_{1}\right)=\pi\left(v_{2}\right)$. However, since $\sigma$ is a proper circular-arc order of $G$, all vertices between $v_{1}$ and $v_{2}$ in $\sigma$ are thus neighbours of $w$, which straightforwardly implies $d_{G}(w)>\Delta$.

Theorem 2. If $n \not \equiv k(\bmod (\Delta+1))$, for all $k \in\{1, \Delta\}$, and $G$ has a maximal clique of size 2, then $G$ is: Class 1 and $(\Delta+2)$-total colourable if $\Delta$ is odd; Type 1 if $\Delta$ is even.

Proof. If $r:=n \bmod (\Delta+1)=0$, we are done by Theorem 1 . If $\Delta \leq 2$, then $G$ is a cycle or a disjoint union of paths and the theorem clearly holds. Hence, we assume that $\Delta \geq 3$ and $r \neq 0$. Let $\sigma:=u_{0}, \ldots, u_{n-1}$ be a proper circular-arc order of $G$, being $\left\{u_{0}, u_{n-1}\right\}$ a maximal clique. Because $\sigma$ is a proper circular-arc order, we have $u_{\Delta} \notin N_{G}\left(u_{0}\right)$ and $u_{n-1-\Delta} \notin N_{G}\left(u_{n-1}\right)$, otherwise $d_{G}\left(u_{0}\right)>\Delta$ or $d_{G}\left(u_{n-1}\right)>\Delta$.

Let $V\left(K_{\Delta+1}\right):=\{0, \ldots, \Delta\}$ and let $\varphi \in\left\{\varphi_{\text {edge }}^{\text {even }}, \varphi_{\text {total }}\right\}$ be the canonical total or edge-colouring of the $K_{\Delta+1}$. The function $\pi: V\left(G^{\prime}\right) \rightarrow V\left(K_{\Delta+1}\right)$ defined by $\pi\left(u_{i}\right):=$ $i \bmod (\Delta+1)$ is clearly a pullback from $G^{\prime}:=G-u_{n-1} u_{0}$ to the $K_{\Delta+1}$ and brings a total or an edge-colouring $\psi$ of $G^{\prime}$ using the same set of colours as $\varphi$. Ergo, we have only to colour $u_{n-1} u_{0}$ in order to complete the proof.

Observe that $\pi\left(u_{n-1}\right)=r-1, \pi\left(u_{n-1-\Delta}\right)=r$, and, since neither $r$ nor $r-1$ is $\Delta$, $\varphi(r, r-1)=(2 r-1) \bmod d=: q$, with $d:=\Delta$ if $\varphi=\varphi_{\text {edge }}^{\text {even }}$, or $d:=\Delta+1+\operatorname{even}(\Delta+1)$ if $\varphi=\varphi_{\text {total }}$. Therefore, as $\pi(v) \neq \Delta$ and $\pi(w) \neq r$ for all $v \in N_{G^{\prime}}\left(u_{0}\right)$ and all $w \in$ $N_{G^{\prime}}\left(u_{n-1}\right)$, the colour $\varphi(0, \Delta)$ is missing at $u_{0}$ and the colour $q$ at $u_{n-1}$. If $q=\varphi(0, \Delta)$, then we assign the colour $q$ to $u_{n-1} u_{0}$ and we are done. Otherwise, since $q \in\{0, \ldots, \Delta\}$, we exchange $\Delta$ and $q$ in the codomain of $\pi$, that is, we redefine $\pi$ so that every vertex which has been mapped by $\pi$ to $\Delta$ is now mapped to $q$ and vice versa. Notice that the images of $u_{0}, u_{n-1-\Delta}$, and $u_{n-1}$ by $\pi$ remain the same, but $\pi\left(u_{\Delta}\right)$ becomes $q$, which now is also a colour missing at $u_{0}$. Then, we colour $u_{n-1} u_{0}$ with $q$.

\section{Final remarks}

Let $\mathcal{A}$ be the class of the proper circular-arc graphs with odd $\Delta$ and a maximal clique of size 2. Overfull graphs in $\mathcal{A}$ can be constructed for $n \equiv 1$ and for $n \equiv \Delta(\bmod (\Delta+1))$ (see Figures 2(a) and 2(b), respectively). Since Theorem 2 can be interestingly used to 
a)

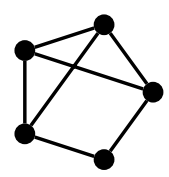

b)

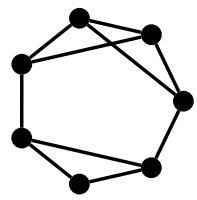

Figure 2. Two overfull graphs in $\mathcal{A}$

show a graph in $\mathcal{A}$ is $S O$ if and only if it is overfull, we conclude proposing the following:

Conjecture. A graph in $\mathcal{A}$ is Class 2 if and only if it is overfull.

\section{References}

Behzad, M. (1965). Graphs and their chromatic numbers. PhD thesis, Michigan State University.

Behzad, M., Chartrand, G., and Cooper Jr., J. K. (1967). The color numbers of complete graphs. J. London Math. Soc., 42:226-228.

Booth, K. S. and Lueker, G. S. (1976). Testing for the consecutive ones property, interval graphs, and graph planarity using pq-tree algorithms. J. Comput. Syst. Sci., 13:335379.

Campos, C. N., Figueiredo, C. M. H., Machado, R., and Mello, C. P. (2012). The total chromatic number of split-indifference graphs. Discrete Math., 312:2690-2693.

Figueiredo, C. M. H., Meidanis, J., and Mello, C. P. (1997). On edge-colouring indifference graphs. Theor. Comput. Sci., 181:91-106.

Figueiredo, C. M. H., Meidanis, J., Mello, C. P., and Ortiz, C. (2003). Decompositions for the edge colouring of reduced indifference graphs. Theor. Comput. Sci., 297:145-155.

Garey, M. R., Johnson, D. S., Miller, G. L., and Papadimitriou, C. H. (1980). The complexity of coloring circular arcs and chords. SIAM J. Algebraic Discrete Methods, $1(2): 216-227$.

Grötschel, M., Lovász, L., and Schrijver, A. (1981). The ellipsoid method and its consequences in combinatorial optimization. Combinatorica, 1(2):169-197.

Hilton, A. J. W. and Johnson, P. D. (1987). Graphs which are vertex-critical with respect to the edge-chromatic number. Math. Proc. Cambridge Philos. Soc., 102:103-112.

Holyer, I. (1981). The NP-completeness of edge-colouring. SIAM J. Comput., 10(4):718720.

McConnell, R. M. (2003). Linear-time recognition of circular-arc graphs. 37:93-147.

Roberts, F. S. (1969). Indifference graphs. In Proc. $2^{\text {nd }}$ Ann Arbor Graph Theory Conference, pages 139-146, Ann Arbor, USA.

Sánchez-Arroyo, A. (1989). Determining the total colouring number is NP-hard. Discrete Math., 78:315-319.

Vizing, V. G. (1964). On an estimate of the chromatic class of a p-graph (in Russian). Diskret. Analiz., 3:25-30.

Vizing, V. G. (1968). Some unsolved problems in graph theory. Russian Math. Surveys, 23:125-141. 